\title{
Lean mass predicts asthma better than fat mass among females
}

\author{
A. Sood*, C. Qualls*, R. Li*, M. Schuyler*, W.S. Beckett ${ }^{\Uparrow}$, L.J. Smith ${ }^{+}$, \\ B. Thyagarajan ${ }^{\S}$, C.E. Lewis ${ }^{f}$ and D.R. Jacobs $\mathrm{Jr}^{\star *}{ }^{\# \#}$ for the CARDIA Investigators
}

ABSTRACT: The obesity phenotype associated with asthma is not known. Our objective was to define the relative contribution of various distributions of fat and lean mass to asthma prevalence.

Data were obtained from 2,525 participants (including 1,422 females) who underwent dualenergy X-ray absorptiometry (DEXA) at the year 20 examination in the Coronary Artery Risk Development in Young Adults (CARDIA) cohort. Total, truncal, arm and leg distributions of fat and lean mass were adjusted to the person's height. Self-reported asthma was the outcome.

Asthma among females was associated with greater total fat mass, arm fat mass, total lean mass, truncal lean mass and arm lean mass. Among males, none of these mass measures were significantly associated with asthma. Among females, the association with asthma was stronger for total lean mass than for total fat mass. Further, among various regional distributions of lean and fat mass in females, truncal lean mass was the strongest predictor.

Total lean mass is more strongly associated with asthma than total fat mass among females. These findings are contrary to the popular perception that excess physiological fat drives the obesity-asthma association. Rather, we hypothesise that ectopic fat within the "lean" tissues drives this association among females.

KEYWORDS: Asthma, dual energy X-ray absorptiometry, fat mass, lean mass

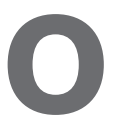
besity is a risk factor for asthma, particularly among females [1-3]. The mechanistic basis for the association between obesity and asthma is unknown and was considered a research priority at a United States National Heart, Lung and Blood Institutes workshop [3]. A major limitation of the obesity-asthma literature is that it has focused on body mass index (BMI) to define obesity. It is now known that a large value for BMI is in fact a collection of various phenotypes, some, but not all, relating to adiposity. It is unclear whether asthma is associated with a specific phenotype of obesity.

Various distributions of fat and skeletal muscle (such as in the trunk, arm and leg) may have varying mechanical and inflammatory properties and consequent health-related effects. For instance, truncal fat may carry a higher risk than arm or leg fat for diseases that constitute the metabolic syndrome [4]. In fact, leg fat may be "metabolically protective" and may mitigate insulin resistance [5]. Further, truncal fat may have greater mechanical impact on forced vital capacity than arm or leg fat, an effect that may be important in asthma pathogenesis [6]. Truncal fat itself is a collection of fat compartments with varying metabolic activity, including visceral, deep abdominal wall subcutaneous and superficial abdominal wall subcutaneous fat. Among the various components of truncal fat, visceral fat is the most strongly correlated with features of the metabolic syndrome [4]. While visceral fat may be a relatively more important source of adiponectin [7], abdominal subcutaneous fat may be a relatively more important source of leptin [8]; these adipokines have opposing associations with asthma $[1,9]$. Furthermore, it has become increasingly apparent that intramuscular fat is strongly associated with insulin resistance [10] and low serum adiponectin concentrations [11] although its relationship with asthma is not known. The objective of this study was to define the relative contributions of various distributions of fat and lean mass to asthma prevalence in males and females separately. Since this is the first study in the literature to evaluate the various distributions of fat and lean mass in relation to asthma, we chose a cross-sectional study design.

\section{AFFILIATIONS}

*Dept of Medicine

\#Clinical Translational Science Center, University of New Mexico School of Medicine, Albuquerque $\mathrm{NM}$,

-Dept of Medicine, Mount Auburn Hospital, Cambridge, MA,

+Dept of Medicine, The Feinberg School of Medicine, Northwestern University, Chicago, IL,

${ }^{\S}$ Dept of Laboratory Medicine and Pathology,

${ }^{*}$ Division of Epidemiology and Community Health, School of Public Health, University of Minnesota Minneapolis, MN, and

${ }^{f}$ Dept of Medicine, University of Alabama at Birmingham, Birmingham, AL, USA.

\#\# Dept of Nutrition, University of Oslo, Oslo, Norway.

CORRESPONDENCE

A. Sood

Division of Pulmonary and Critical Care Medicine

University of New Mexico Health Sciences Center School of Medicine Dept of Medicine

1 University of New Mexico

MSC 105550

ACC 5

Albuquerque

NM 87131

USA

E-mail: asood@salud.unm.edu

Received

Dec 072009

Accepted after revision:

May 042010

First published online:

June 042010 


\section{METHODS}

\section{Study design}

This was a cross-sectional data analysis from the year 20 examination of the prospective Coronary Artery Risk Development in Young Adults (CARDIA) cohort. During 1985-1986, CARDIA randomly recruited 5,115 black and white males and females, 18-30 yrs of age, from the general population of Birmingham, AL, Chicago, IL and Minneapolis, $\mathrm{MN}$, and from the membership of the Oakland KaiserPermanente Health Plan in Oakland, CA (USA). Follow-up examinations were completed 2, 5, 7, 10, 15 and 20 yrs later. Detailed methods, instruments and quality control procedures are described at the CARDIA website [12] and in other published reports $[13,14]$. Retention of CARDIA participants has been excellent, as 3,549 persons were examined at the Year 20 examination, constituting $72 \%$ of the survivors from the baseline cohort. Of those examined at year 20, 2,705 participants $(76.2 \%)$ underwent dual-energy X-ray absorptiometry (DEXA) examination. After excluding those with former asthma $(n=180)$ at year 20, data on 2,525 participants were analysed. Participants with former asthma were excluded in the primary analysis to obtain the greatest discrimination between those with current asthma and without asthma. Alternate analyses were performed by separately combining participants with former asthma with either of the two groups with current or never asthma. Self-reported information was obtained from all participants using standardised questionnaires. Height and weight were measured by certified technicians using standardised equipment with participants wearing light clothing and no shoes.

\section{DEXA}

DEXA (Hologic QDR 4500; Hologic, Bedford, MA, USA) was used to determine fat mass and lean mass (i.e. nonfat and nonbone soft tissue content) for the whole body and its regions (i.e. trunk, arms and legs). DEXA-assessed lean mass primarily comprises of skeletal muscle and visceral mass but includes fat deposits present within and around muscle and viscera. Analogous to the commonly used BMI, all DEXA-assessed mass measures were adjusted for the person's height [15]. Additional details about DEXA measurements and quality control are provided in the supplementary material. As discussed in the depository, we noted no evidence of selection bias on the obesity-asthma association with respect to DEXA measurement at year 20 examination.

\section{Outcome}

Participants with self-reported current asthma were compared to those without asthma. Current asthma was defined as participant self-report of healthcare provider-diagnosed asthma at any time plus either presence of current asthma symptoms during the previous 12 months or a validated participant report of current use of asthma medication(s). This definition is more stringent than in most epidemiological studies, where provider diagnosis is the sole criterion for defining asthma [16]. Validation of medication use was performed whenever possible by direct examination of the medication containers by study personnel. Those who never reported having asthma or taking asthma medications at any of the examinations were included in the referent group. Former asthma was similarly defined as participant self-report of provider-diagnosed asthma at any time, plus absence of both current asthma symptoms during the previous 12 months and current use of asthma medication(s).

\section{Covariates}

All multivariate models include the following covariates: selfreported smoking status, race, age, study centre at which the DEXA was performed and physical activity; all of which were measured at the Year 20 examination. Smoking status was treated as a categorical variable, comprising current, former and never-smokers. The frequency of participation in a range of specific heavy- and moderate-intensity physical activities in the previous year was obtained from a validated questionnaire [17]. Data were converted into a weighted physical activity score (in exercise units), using the methods of FoLSOM et al. [18]. The score was logarithmically transformed because of its nonnormal distribution.

\section{Statistical analysis}

Tertiles of predictor variables were studied since several variables were not normally distributed, despite logarithmic transformation. Multivariate logistic regression analyses were used in males and females separately (since our previous studies had demonstrated the obesity-asthma association only in females) [1, 2]. The effect of total lean and fat mass measures on asthma was separately studied after adjusting for covariates and is presented as minimally adjusted models. Each mass measure was then adjusted for the other, presented as a full model, to obtain the stronger predictor. Similar analyses for additional components of fat and lean mass were performed, as discussed in the supplementary information. Multiplicative interactions of sex on the associations between various predictor measures and asthma status were also studied. A two-sided $\mathrm{p}$-value of $<0.05$ was considered statistically significant for all tests. All statistical analyses were performed using the Statistical Analysis Software (SAS) package version 9.1 (SAS Institute, Cary, NC, USA). All subjects gave informed consent for their participation in the study. This study was approved by the Institutional Review Boards at University of New Mexico, Albuquerque, NM and at each of the CARDIA study sites.

\section{RESULTS \\ Participant characteristics}

Data on 2,525 subjects (1,103 males and 1,422 females) were analysed. Almost twice as many females (146 or $10.3 \%$ ) as males (59 or $5.3 \%$ ) had asthma. As expected, males had greater lean mass and lesser fat mass than females in all regions of the body $(\mathrm{p}<0.001$, table 1$)$. For every unit of total fat mass, males had approximately twice as much total lean mass as females. A greater proportion of fat mass was distributed in the trunk in males, as compared to females.

\section{Fat and lean mass measures associated with asthma}

In bivariate analyses (table 1), females with asthma had greater fat and lean mass than females without asthma, irrespective of the region of distribution ( $p \leqslant 0.02$ for all analyses). Interestingly, males with asthma had lower mass measures than males without asthma, but these differences were not significant.

In multivariate analyses, total fat mass and arm fat mass were both positively associated with asthma among females 


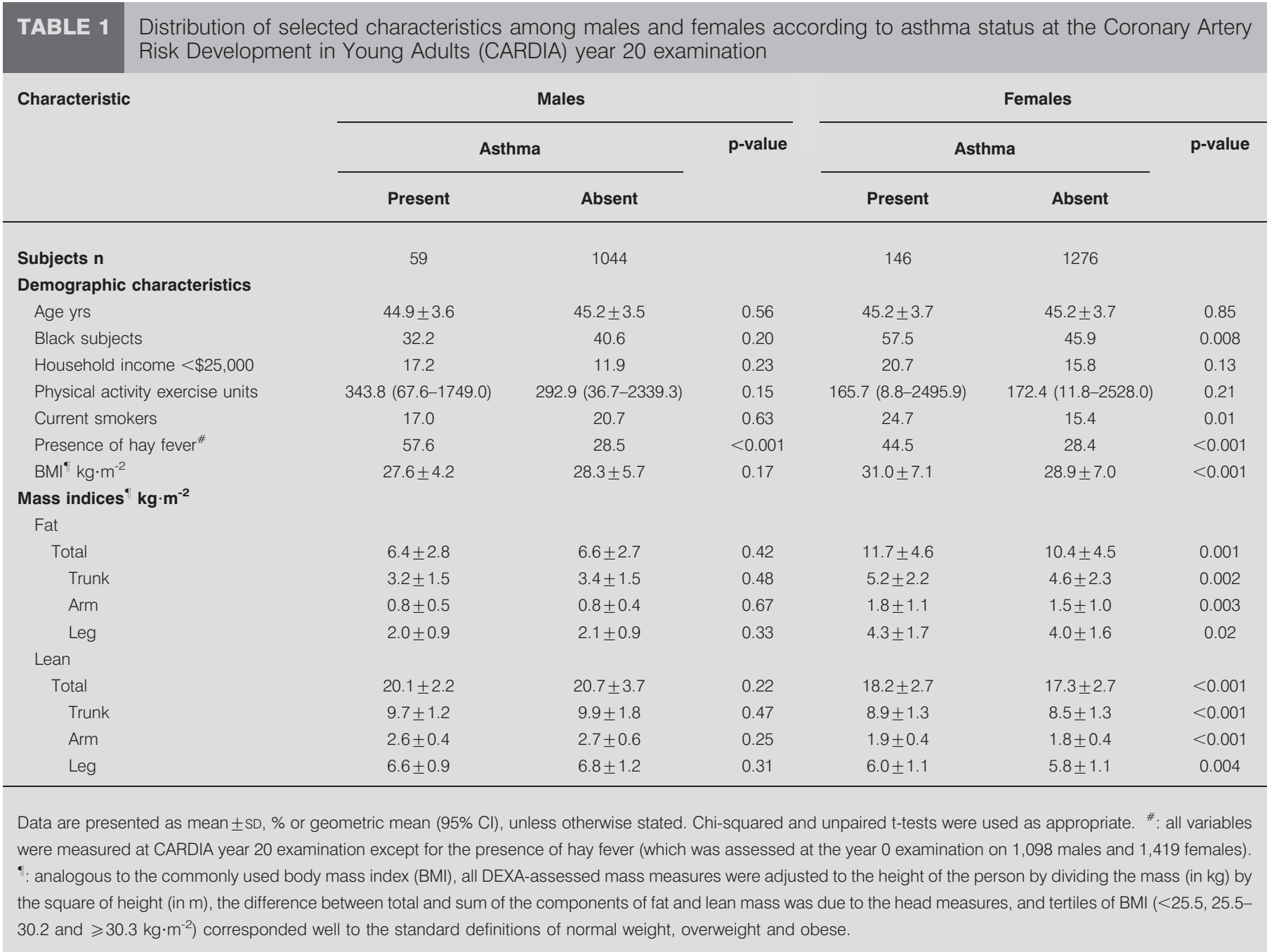

$(p \leqslant 0.02$ for both analyses; weak sex interactions with $p \leqslant 0.13$ for both analyses; table 2). Surprisingly, truncal fat mass was not significantly associated with asthma in either sex in the multivariate analysis. Total lean mass, truncal lean mass and arm lean mass were positively associated with asthma in females ( $p \leqslant 0.004$ for all analyses; sex interactions with $p \leqslant 0.11$ for all analyses). Neither fat nor lean mass measures were significantly associated with asthma among males.

\section{Relative contributions of fat and lean mass measures towards asthma}

To better understand the relative contributions of fat and lean mass measures towards the odds for asthma, we first performed correlational analysis between corresponding fat and lean mass measures (table 3). The overall correlations were stronger among females than males. However, formal tests of collinearity suggested that the corresponding fat and lean mass variables were not collinear. Further, in order to quantify the relative contributions of total fat mass and total lean mass to the odds for asthma among females, we performed multivariate logistic regression analyses (table 4). In the minimally adjusted models, total lean mass was found to be a slightly stronger predictor of asthma among females than total fat mass. Adjusting total lean mass for total fat mass (in the full model) did not eliminate the association of total lean mass with asthma among females. On the other hand, adjusting total fat mass for total lean mass reduced the association of total fat mass with asthma among females to a nonsignificant level. This suggests that lean mass is a stronger predictor for asthma among females than fat mass and that the lean mass-asthma association is not confounded by fat mass.

We further analysed the relative contribution of the various distributions of fat and lean mass to the odds for asthma in females in table 5. Among various measures of lean and fat mass studied, truncal lean mass and arm fat mass were found to be the strongest predictors of asthma respectively among females in the minimally adjusted models. After full adjustment, truncal lean mass remained a strong predictor of asthma among females but arm fat mass did not. However, leg lean mass was inversely associated with asthma among females. Truncal fat mass was, however, not associated with asthma among females.

\section{Alternate analytic strategies}

Alternate analytic strategies are discussed in the supplementary material and show no effect of atopy, menopause or race on the described associations. We reanalysed the data, 


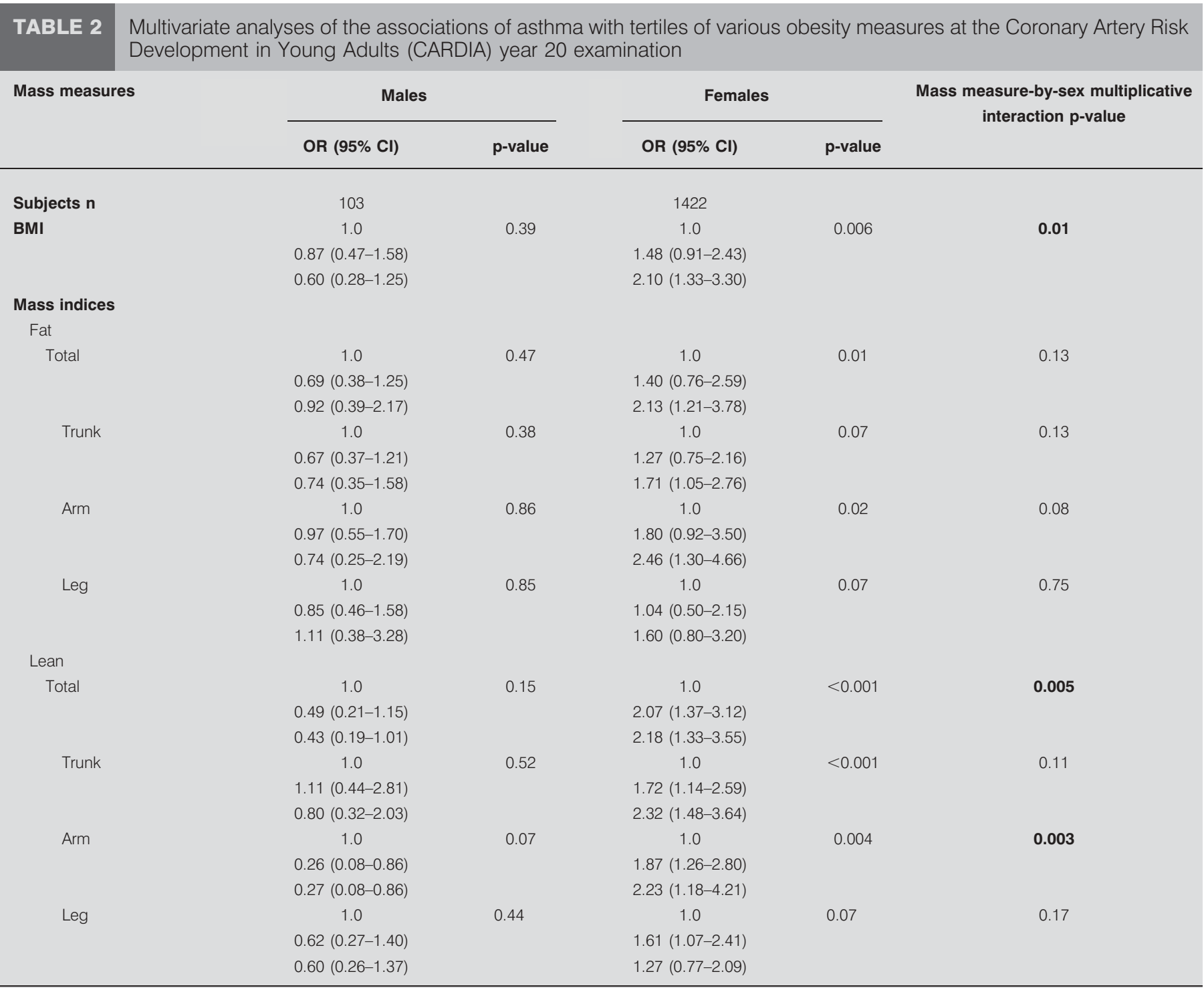

All analyses were adjusted for age, race, clinical centre, smoking status and log-transformed physical activity score. All mass measures were height-adjusted by dividing the mass (in $\mathrm{kg}$ ) by the square of height (in $\mathrm{m}$ ). Lowest tertile for each measure was used as referent value. Similar results were obtained after additional adjustment for atopic status (as evidenced by self-reported hay fever) at baseline (year 0 ) examination. BMl: body mass index. Bold indicates statistically significant $\mathrm{p}$-values. $\mathrm{n}=2,525$.

including participants with former asthma among either of the two groups with current asthma and never-asthma and obtained similar results. Principal components analysis was performed and showed that no combination of regional fat and lean mass measures was superior to truncal lean mass alone in predicting asthma in females.

\section{DISCUSSION}

We found that increased total lean mass was associated with higher odds for asthma only among females in this crosssectional analysis nested within the prospective CARDIA study. This association was stronger than the association of total fat mass with asthma among females. Among all the regional fat and lean mass measures, truncal lean mass was the strongest predictor. However, neither fat nor lean mass measures were associated with asthma among males. These findings are contrary to the popular perception that excess physiological fat drives the obesity-asthma association in females.

Why might greater lean mass be associated with higher odds for asthma among females? DEXA-assessed "lean" mass is not entirely fat-free but includes the smaller and highly metabolically active ectopic fat within the skeletal muscle and viscera. SUTHERLAND et al. [19], in their study of lung function, suggested that, while lean mass was a measure of muscle bulk in males, excess lean mass in females was a measure of obesity. It is therefore possible that excess ectopic fat may explain our findings in obese females.

Intramuscular fat infiltration, also described in lay terms as "marbling of muscle", is more pronounced in females than males, independent of overall obesity [20, 21]. Consistent with these observations, our data demonstrate that positive 


\begin{tabular}{|c|c|c|c|}
\hline TABLE 3 & \multicolumn{3}{|c|}{$\begin{array}{l}\text { Correlation coefficients between dual-energy } \\
\text { X-ray absorptiometry-assessed fat and lean } \\
\text { mass measures in males and females at the } \\
\text { Coronary Artery Risk Development in Young } \\
\text { Adults (CARDIA) year } 20 \text { examination }\end{array}$} \\
\hline \multicolumn{2}{|c|}{ Fat and lean mass components } & Males & Females \\
\hline \multicolumn{2}{|l|}{ Subjects $n$} & 1103 & 1422 \\
\hline \multicolumn{2}{|c|}{ Total fat verus total lean mass } & 0.52 & 0.77 \\
\hline \multicolumn{2}{|c|}{ Truncal fat versus truncal lean mass } & 0.53 & 0.73 \\
\hline \multicolumn{2}{|c|}{ Arm fat versus arm lean mass } & 0.38 & 0.67 \\
\hline \multicolumn{2}{|c|}{ Leg fat versus leg lean mass } & 0.53 & 0.72 \\
\hline
\end{tabular}

correlations between lean mass and fat mass measures are stronger among obese females than obese males (table 3). Further, intramuscular fat is associated with high systemic leptin and low systemic adiponectin concentrations [11, 22-25] and increased expression of tumour necrosis factor (TNF)- $\alpha$ [22], changes possibly associated with asthma [1, 9, 23].

Additionally, truncal lean mass includes visceral fat components that may be associated with asthma. Visceral fat, constituting only about $10 \%$ of total fat, surrounds and infiltrates internal organs in the intrathoracic, intra-abdominal and intrapelvic compartments. Our study is unable to show whether a specific component of visceral fat (such as epicardial or hepatic fat) drives the obesity-asthma association in females. Nevertheless, pro-inflammatory adipokines expressed by visceral fat components may have direct access to portal and systemic circulation [24], accounting for the disproportionately greater inflammatory effect of visceral fat relative to its small size. Furthermore, like intramuscular fat, visceral fat may be associated with high systemic leptin and TNF- $\alpha$ and low systemic adiponectin concentrations [25-29], changes again possibly associated with asthma [1, 9, 22]. Also, truncal mass (of which there is disproportionately more lean than fat mass) may have direct mechanical effects on diaphragmatic position and movement and chest wall recoil that may increase airway responsiveness [30].

Unlike truncal lean mass, which was positively associated with asthma among females, we report that leg lean mass was inversely associated with asthma among females. One possible explanation is that skeletal muscle in different regions may have different metabolic and inflammatory effects [31]. However, the bases for these differences remain unknown.

Our study also suggests that lean mass may be a better predictor than fat mass for asthma among females. A plausible explanation is that the smaller ectopic fat depots (components of DEXA-assessed lean mass) may be more important from the inflammatory standpoint than the larger, physiological fat depots.

Furthermore, why might arm fat (which constitutes approximately $13 \%$ of total fat) be a better predictor for asthma among females than other, larger fat compartments in the body (table 5 and supplementary table E3)? Arm fat may have a different metabolic profile compared to either truncal fat or leg fat [32]. Relative to other fat compartments, leg fat may enhance serum adiponectin concentrations and be "metabolically benign" [31]. However, truncal fat is not a uniform compartment but includes two subcomponents with varying metabolic activity: superficial abdominal subcutaneous fat that is metabolically benign and deep abdominal subcutaneous fat that is metabolically active (similarly to visceral fat) [33]. However, since DEXA cannot separate these two subcomponents, the net effect of truncal fat mass on asthma in females may be neutralised.

We also note a sexual dimorphism of specific lean mass measures with respect to asthma, since the odds ratios are directed oppositely in males and females. This is not due to the smaller height in females, since height-adjusted mass indices were used for these analyses. Possible explanations include the effect of sex hormones, gonadotrophic hormones or sex hormone-binding globulin, sex-specific effects of adipokines, sex-specific differences in adiponectin isoform distribution and intramuscular lipid deposition.

Finally, participants with asthma at CARDIA year 20 examination were mostly of intermittent or mildly persistent severity.

TABLE 4 Multivariate analysis showing a stronger association of total lean mass than total fat mass on asthma in females at the Coronary Artery Risk Development in Young Adults (CARDIA) year 20 examination

\begin{tabular}{|c|c|c|c|c|}
\hline \multirow{2}{*}{$\begin{array}{l}\text { Fat and lean mass } \\
\text { component }\end{array}$} & \multicolumn{2}{|c|}{ Minimally adjusted model } & \multicolumn{2}{|c|}{ Full model } \\
\hline & $\begin{array}{l}\text { OR for third tertile versus } \\
\text { first tertile }(95 \% \mathrm{CI})\end{array}$ & Overall p-value & $\begin{array}{l}\text { OR for third tertile versus } \\
\text { first tertile }(95 \% \mathrm{Cl})\end{array}$ & Overall p-value \\
\hline Total lean mass & $2.18(1.33-3.55)$ & $<0.001$ & $1.87(1.04-3.36)$ & 0.03 \\
\hline Total fat mass & $2.13(1.21-3.78)$ & 0.01 & $1.46(0.77-2.77)$ & 0.52 \\
\hline
\end{tabular}




\begin{tabular}{|c|c|c|c|c|}
\hline 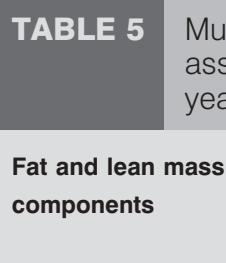 & $\begin{array}{l}\text { te analysis of six lean and fat } m \\
\text { on with asthma among females } \\
\text { xamination }\end{array}$ & $\begin{array}{l}\text { omponents s } \\
\text { 122) at the Col }\end{array}$ & $\begin{array}{l}\text { ig that truncal lean mass has th } \\
\text { y Artery Risk Development in You }\end{array}$ & $\begin{array}{l}\text { ongest positive } \\
\text { Adults (CARDIA) }\end{array}$ \\
\hline $\begin{array}{l}\text { Fat and lean mass } \\
\text { components }\end{array}$ & OR for the third tertile only $(95 \% \mathrm{Cl})$ & Overall $p$-value & OR for the third tertile only $(95 \% \mathrm{Cl})$ & Overall $p$-value \\
\hline Leg fat mass & $1.60(0.80-3.20)$ & 0.07 & $0.83(0.31-2.21)$ & 0.41 \\
\hline Truncal lean mass & $2.32(1.48-3.64)$ & $<0.001$ & $2.81(1.39-5.69)$ & 0.02 \\
\hline Arm lean mass & $2.23(1.18-4.21)$ & 0.004 & $1.79(0.82-3.91)$ & 0.22 \\
\hline Leg lean mass & $1.27(0.77-2.09)$ & 0.07 & $0.40(0.19-0.84)$ & 0.02 \\
\hline
\end{tabular}

To assess the relative strength of association of various components of fat and lean mass on asthma, we show the effect of each component in separate logistic regression analyses in the minimally adjusted model and each of their effect after adjustment for all other components in the full model. Each row presents the third tertile compared to the first. In addition, both minimally adjusted and full models were adjusted for covariates: age, race, clinical centre, smoking status, and log-transformed physical activity score. Similar results were obtained after additional adjustment for atopic status (as evidenced by self-reported hay fever) at baseline (year 0) examination. Bold indicates statistically significant $p$-values.

Thus, systemic steroid intake for the treatment of asthma was reported by only two participants at that examination, both being males. Therefore, we do not think our findings can be explained by the effect of systemic steroids on the repartition of fat and lean tissues.

The strengths of our study include its sex-specific stratified analysis, well-defined study population set within a cohort structure, adjustment for physical activity and use of DEXA data to assess regional body composition. Instead of using absolute mass obtained DEXA, we have adjusted all mass measures for height, which may allow for better comparison of individuals of different sizes [34]. Furthermore, the study results generate innovative hypotheses regarding the mechanistic basis for the obesity-asthma association.

Nevertheless, DEXA itself has some limitations. Current DEXA software assumes a constant water content of lean mass, but whether this is true in fluid-overloaded obese populations is unknown. DEXA inaccurately assesses ectopic fat as lean mass. Its accuracy in the obese may also be adversely affected by the effect of increased body thickness on beam hardening and increased body size falling outside the scan range or scanner weight limit. Although whole-body region DEXA strongly correlates with site-specific DEXA, the measurement may provide somewhat different estimates. Several limitations of DEXA may be overcome in future studies of the role of the lipid content of skeletal muscle and viscera in asthma by using computerised tomography, magnetic resonance spectroscopy or histopathological techniques.

The study has some additional limitations as well. Atopic status was measured not at year 20 but at year 0 examinations, using self-report. The results of this study may not be generalisable to populations other than black and white subjects. This study will not be able to evaluate objectively defined asthma phenotypes. Self-reported asthma definitions are well accepted in the epidemiological literature but may result in misclassification bias [16, 35]. However, this bias is likely to be nondifferential between obese and nonobese populations [35]. Nondifferential misclassification biases the estimate of association towards the null value and is unlikely to produce a spurious effect [16]. Finally, this is an observational, cross-sectional study which allows for hypothesis generation but does not prove causality or the direction of association. However, prior prospective studies have suggested that obesity precedes asthma and not vice versa [16].

Our findings that increased total lean mass is more strongly associated with asthma than fat mass among females are contrary to the popular perception that excess physiological fat drives the obesity-asthma association. Rather, we hypothesise that ectopic fat within the lean tissues drives this association among females. Our cross-sectional results do allow for innovative hypothesis generation in the field of obesity and asthma. However, our hypotheses would need further confirmation with longitudinal study designs and/or use of more sophisticated techniques of measuring ectopic fat, such as computed tomography and magnetic resonance spectroscopy. If true, decreasing ectopic fat mass, and not simply increasing the fat-free lean mass or decreasing physiological fat mass, would be a reasonable goal in the prevention and treatment of asthma among obese females.

\section{STATEMENT OF INTEREST}

None declared.

\section{ACKNOWLEDGEMENTS}

The authors wish to thank R. Kalhan and M. Carnathon (both Northwestern University, Chicago, IL, USA) for their careful critiques of the manuscript.

\section{REFERENCES}

1 Sood A, Cui X, Qualls C, et al. Association between asthma and serum adiponectin concentration in women. Thorax 2008; 63: 877-882. 
2 Sood A, Qualls C, Arynchyn A, et al. Obesity-asthma association: is it explained by systemic oxidant stress? Chest 2009; 136: 1055-1062.

3 Weiss ST, Shore SA. Obesity and asthma: directions for research. Am J Respir Crit Care Med 2004; 169: 963-968.

4 Bergman RN, Kim SP, Hsu IR, et al. Abdominal obesity: role in the pathophysiology of metabolic disease and cardiovascular risk. Am J Med 2007; 120: Suppl. 1, S3-S8.

5 Yim JE, Heshka S, Albu JB, et al. Femoral-gluteal subcutaneous and intermuscular adipose tissues have independent and opposing relationships with CVD risk. J Appl Physiol 2008; 104: 700-707.

6 Ding DJ, Martin JG, Macklem PT. Effects of lung volume on maximal methacholine-induced bronchoconstriction in normal humans. J Appl Physiol 1987; 62: 1324-1330.

7 Swarbrick MM, Havel PJ. Physiological, pharmacological, and nutritional regulation of circulating adiponectin concentrations in humans. Metab Syndr Relat Disord 2008; 6: 87-102.

8 Montague CT, Prins JB, Sanders L, et al. Depot- and sex-specific differences in human leptin mRNA expression: implications for the control of regional fat distribution. Diabetes 1997; 46: 342-347.

9 Sood A, Camargo Jr CA, Ford ES. Association between leptin and asthma in adults. Thorax 2006; 61: 300-305.

10 Goodpaster BH, Thaete FL, Simoneau JA, et al. Subcutaneous abdominal fat and thigh muscle composition predict insulin sensitivity independently of visceral fat. Diabetes 1997; 46: 1579-1585.

11 Weiss R, Dufour S, Groszmann A, et al. Low adiponectin levels in adolescent obesity: a marker of increased intramyocellular lipid accumulation. J Clin Endocrinol Metab 2003; 88: 2014-2018.

12 Coronary Artery Risk Development in Young Adults. Exam materials. www.cardia.dopm.uab.edu/ex_mt.htm Date last accessed: January 24, 2007. Date last updated: 2010.

13 Friedman GD, Cutter GR, Donahue RP, et al. CARDIA: study design, recruitment, and some characteristics of the examined subjects. J Clin Epidemiol 1988; 41: 1105-1116.

14 Hughes GH, Cutter G, Donahue R, et al. Recruitment in the Coronary Artery Disease Risk Development in Young Adults (Cardia) Study. Control Clin Trials 1987; 8: 4 Suppl., 68S-73S.

15 Kenny AM, Dawson L, Kleppinger A, et al. Prevalence of sarcopenia and predictors of skeletal muscle mass in nonobese women who are long-term users of estrogen-replacement therapy. J Gerontol A Biol Sci Med Sci 2003; 58: M436-M440.

16 Ford ES. The epidemiology of obesity and asthma. J Allergy Clin Immunol 2005; 115: 897-909.

17 Jacobs DR Jr, Hahn LP, Haskell WL, et al. Validity and reliability of a short physical activity history: CARDIA and the Minnesota Heart Health Program. J Cardiopulm Rehabil 1989; 9: 448-459.

18 Folsom AR, Jacobs DR Jr, Caspersen CJ, et al. Test-retest reliability of the Minnesota Leisure Time Physical Activity Questionnaire. J Chronic Dis 1986; 39: 505-511.
19 Sutherland TJ, Goulding A, Grant AM, et al. The effect of adiposity measured by dual-energy X-ray absorptiometry on lung function. Eur Respir J 2008; 32: 85-91.

20 Miljkovic-Gacic I, Wang X, Kammerer CM, et al. Fat infiltration in muscle: new evidence for familial clustering and associations with diabetes. Obesity (Silver Spring) 2008; 16: 1854-1860.

21 Forsberg AM, Nilsson E, Werneman J, et al. Muscle composition in relation to age and sex. Clin Sci (Lond) 1991; 81: 249-256.

22 Mingrone G, Rosa G, Di Rocco P, et al. Skeletal muscle triglycerides lowering is associated with net improvement of insulin sensitivity, TNF- $\alpha$ reduction and GLUT4 expression enhancement. Int J Obes Relat Metab Disord 2002; 26: 1165-1172.

23 Berry MA, Hargadon B, Shelley M, et al. Evidence of a role of tumor necrosis factor $\alpha$ in refractory asthma. N Engl J Med 2006; 354: 697-708.

24 Bjorntorp P. "Portal" adipose tissue as a generator of risk factors for cardiovascular disease and diabetes. Arteriosclerosis 1990; 10: 493-496.

25 Finck BN, Johnson RW. Tumor necrosis factor- $\alpha$ regulates secretion of the adipocyte-derived cytokine, leptin. Microsc Res Tech 2000; 50: 209-215.

26 Trujillo ME, Sullivan S, Harten I, et al. Interleukin-6 regulates human adipose tissue lipid metabolism and leptin production in vitro. J Clin Endocrinol Metab 2004; 89: 5577-5582.

27 Bruun JM, Lihn AS, Verdich C, et al. Regulation of adiponectin by adipose tissue-derived cytokines: in vivo and in vitro investigations in humans. Am J Physiol Endocrinol Metab 2003; 285: E527-E533.

28 Cinti S, Mitchell G, Barbatelli G, et al. Adipocyte death defines macrophage localization and function in adipose tissue of obese mice and humans. J Lipid Res 2005; 46: 2347-2355.

29 Bertin E, Nguyen P, Guenounou M, et al. Plasma levels of tumor necrosis factor- $\alpha$ (TNF- $\alpha$ ) are essentially dependent on visceral fat amount in type 2 diabetic patients. Diabetes Metab 2000; 26: 178-182.

30 Wang LY, Cerny FJ, Kufel TJ, et al. Simulated obesity-related changes in lung volume increases airway responsiveness in lean, nonasthmatic subjects. Chest 2006; 130: 834-840.

31 Olsen DB, Sacchetti M, Dela F, et al. Glucose clearance is higher in arm than leg muscle in type 2 diabetes. J Physiol 2005; 565: 555-562.

32 Van Pelt RE, Evans EM, Schechtman KB, et al. Contributions of total and regional fat mass to risk for cardiovascular disease in older women. Am J Physiol Endocrinol Metab 2002; 282: E1023-E1028.

33 Walker GE, Verti B, Marzullo P, et al. Deep subcutaneous adipose tissue: a distinct abdominal adipose depot. Obesity (Silver Spring) 2007; 15: 1933-1943.

34 Kyle UG, Genton L, Gremion G, et al. Aging, physical activity and height-normalized body composition parameters. Clin Nutr 2004; 23: 79-88

35 Aaron SD, Vandemheen KL, Boulet LP, et al. Overdiagnosis of asthma in obese and nonobese adults. CMAJ 2008; 179: 1121-1131. 